\title{
Severely atrophied jaws: A case report of full mouth rehabilitation using basal and cortical implants
}

\author{
T. K Pal ${ }^{1}$, Abhijit Mahata ${ }^{2}$, Goutam Das ${ }^{3, *}$ \\ ${ }^{1}$ Professor, ${ }^{2}$ Consultant, ${ }^{3}$ Senior Lecturer, ${ }^{3} \mathrm{Ph}$.D. Scholar, ${ }^{3}$ Dept. of Periodontics, ${ }^{1} \mathrm{JIS}$ University, West Bengal, ${ }^{2}$ Restorative and \\ Implants Director Smile and Profile Clinic, ${ }^{3}$ Guru Nanak Institute Dental Sciences and Research, JIS University, West Bengal, \\ India
}

*Corresponding Author:

Email: drgoutamgddc@gmail.com

\begin{abstract}
Patients with highly atrophied jaws pose a serious clinical problem for construction of removal denture or surgical placement of implant. They sometime are in need of undergoing delicate surgical interventions for gaining vertical bone height with/without sinus lift. It requires expensive bone graft substitutes and barrier membranes in the hand of an expert. A long period of time for bone healing is an additional enigma. After the jaws are made ready, the implants are surgically inserted into the bone and a period of another 4-6 months waiting is inevitable. The long waiting period and the expenses for adjuvant surgical treatments are not appreciated by the patients. The present article deals with a patient with highly atrophied jaws for full mouth rehabilitation with basal and cortical implants.
\end{abstract}

Keywords: Jaw atrophy, Bone augmentation, Cortical implants, Full mouth rehabilitation, Strategic implantology.

\section{Introduction}

Placement of conventional implant is usually made with in sufficient amount of alveolar bone which demands 4-6 months of waiting period for healing as per Branemark's concept. ${ }^{1}$ After the healing period, the implant gets attached to the bone through osseo integration and becomes able to withstand the masticatory load. ${ }^{1,2}$ With this, generally, prosthetic loading becomes delayed due to lack of achieving desired implant stability. The situation becomes more complicated in terms of non-availability of sufficient supply of bone for implant placement. ${ }^{3}$ This necessitates precise and expensive adjuvant bone augmentation surgery, ${ }^{4}$ and sometimes sinus lifting. ${ }^{5}$ Jaws are sometimes found so severely atrophied ("no bone situation") that even sophisticated adjuvant surgical procedures are not possible to perform, and thus, atrophic jaws become labeled as 'contraindicated' for implant therapy. Unless strong primary stability is achieved the immediate loading is thought to be far away from reality. ${ }^{6,7}$

In contrast to Branemark's philosophy, the 'Strategic Implantology' offers an unique opportunity to place the specifically designed implants into the cortical and basal bone without relying on the cancellous alveolar bone. ${ }^{8}$ Since the strong cortical and basal bones do not undergo resorption following tooth extraction, it is taken into account as a very stable bone. If the implants are fixed to this bone, a very powerful primary stability is achieved. ${ }^{8-10}$ Here lies the clinical advantage of "strategic implantology" over the Branemark's system. This implant system is now gaining popularity amongst clinicians. ${ }^{11}$

The present case report shows how with flapless approach, the single-piece basal and cortical implants were surgically inserted in various strategic locations of jaws and sphenoid bones, and then functionally loaded with mandibular and maxillary cement retained hybrid prosthesis.

\section{Case Report}

A 68years old woman reported with her edentulous jaws which were severely atrophic. Her chief complaints were the pain and instability of removable mandibular full prosthesis which she had been using for last couple of years with lot of difficulties and thereby, inability to eat property. On intraoral examination. it was revealed that the patient had severely atrophied maxilla and mandibular jaws. There was tenderness on digital pressure on the mid-ridge of mandible at the premolars and first molars areas due to proximity of inferior dental nerve. There was no significant medical history; she had normal gait and stature. The option for conventional removable upper \& lower complete denture was not possible because of severe atrophy of lower jaw. The conventional implant therapy with vertical bone augmentation procedures would have been very time consuming and patient did not accept it. The only option left was with the strategic implants which required only cortical and basal bones of the jaws. The patient accepted this treatment option. Therefore, it was decided to go for cortical \& basal implants.

A complete routine blood examination revealed no significant findings. Panoramic radiography was done and severe atrophy of jaws was noticed in all segments of the jaws (Fig. 1). A 3D model was also made (Fig. 2) for virtual assessment of the severity of atrophy and also to assess the number of implants required in each jaw. This case needed 18 implants (BECES ${ }^{\mathrm{TM}}, \mathrm{BCS}^{\mathrm{TM}} \&$ 


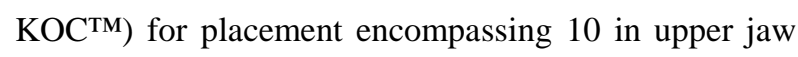
and 8 in lower jaw in various strategic locations.

Under local infiltration anesthesia using $2 \%$ Lignocaine with 1:80,000 Adrenalines Hydrochloride (Lignox A) all the implants were surgically inserted following the rules of "supporting polygon". The supporting polygon denotes, in this context, a 3dimensional spatial concept of a semi-circular bridge construction where the occlusal contacts are made to retain within the polygon formed by drawing imaginary lines of load transmitting threads of the implants at one side (basal bone $/ 2^{\text {nd. }}$ Or $3^{\text {rd }}$ cortical) and the points of their entries through the crest of the alveolar bone $\left(1^{\text {st }}\right.$ cortical) on the other side. The prime positioning of implants in the jaws, as per the supporting polygon, are at the corners or at the end in a semi-circular construction. The anatomical locations of implants in the area of canines and the second molars of both the jaws are known as "strategic positions". ${ }^{12,13}$ The rest of the implants are placed within the polygonal geometry. In each case the implant was dipped into 5\% Betadine solution and then surgically placed in strategic locations of the jaw under flapless procedure into the basal bone, $2^{\text {nd }}$ and $3^{\text {rd }}$ cortical bones as per the protocol (Table 1) (Fig. 3). ${ }^{12}$ Drilling of highly mineralized cortical bones and densely thick trabeculated basal bone of intraforaminal area of symphysis of mandible were made by surgical micromotor at about $38,000-40,000 \mathrm{rpm}$. The tough and highly mineralized cortical bones of maxillofacial-cranial regions can only be cut/osteotomized with this range of speed as against 1000-2500 rpm with Physio-dispenser in conventional implantology. External irrigation is of no use; internal irrigation through long drilling tools are not available. It is said that the slurry produced by the mixture of blood and bone dust makes a coolant paste to some extent.

Once the implants were placed, care was taken so that the implant abutments were at right angle to the surface of the ridge by bending of the implants at their necks. Thereafter, the mandibular and maxillary impressions were made using addition silicone impression materials. It was then followed by recording of provisional jaw relation by wax impregnated with Aluminium powder. A panoramic radiograph was taken for record of the implant placement and in the afternoon of $1^{\text {st }}$ day, routine prescription was made for antibiotics and anti-inflammatory drugs with necessary instructions of diet and oral hygiene care.

Metal framework was made overnight and necessary adjustment was done on metal framework in the patient's mouth during successful try-in (Fig. 4) on $2^{\text {nd }}$ day morning. The occlusal wax rims were made over the metal framework and final vertical dimension of occlusion was recorded. Cusp-free acrylic teeth were setup into the wax rim extending posteriorly up to first molars keeping in mind the basic occlusal plan of 'supporting polygon' for strategic implantology (Fig. 5). ${ }^{12,13}$ In the afternoon hours of 2 nd day, final try-in of metal frame with acrylic teeth set in wax was done with special attention of keeping increased overjet and shallow overbite so that the front teeth should not come in contact in any static or dynamic movement of mandibular excursions. The metal framework with teeth setup was sent to the laboratory for final acrylization trimming and polishing. On the $3^{\text {rd }}$ day morning, all the implants were functionally loaded with both mandibular and maxillary hybrid dentures using Glass Ionomer Cement (Fuji) (Figs. 6\&7). The occlusion was checked at specific points on canines and premolars on both the sides conducive to supporting polygon. ${ }^{8,12,13}$ The patient was asked to follow the post-treatment instructions and recalled after 7 days for general check-up. It took about 50 hours for completion of the procedure from surgery to delivery of hybrid denture. On recall visit, the occlusion was checked using proper bite paper strips on the particular locations of canines, premolars and molars. Final points of contacts according to supporting polygon were establishedon denture. The patient was advised to keep on coming every month for a period of 6 months for check-up and monitoring.

Table 1: Different denominations of cortical bones

\begin{tabular}{|l|l|l|l|}
\hline & \multicolumn{1}{|c|}{ 1st.cortical } & \multicolumn{1}{c|}{ 2nd.cortical } & \multicolumn{1}{|c|}{ 3rd.cortical } \\
\hline Maxilla & Crestal cortical & $\begin{array}{l}\text { Nasal floor, Sinus floor, } \\
\text { Palatal bone, distal cortical of } \\
\text { maxillary pterigoid. }\end{array}$ & $\begin{array}{l}\text { Zygomatic bone, } \\
\text { Pterigoid plate of } \\
\text { sphenoid bone }\end{array}$ \\
\hline Mandible & Crestal cortical & $\begin{array}{l}\text { Lingual cortical, Buccal } \\
\text { cortical }\end{array}$ & Does not exist \\
\hline
\end{tabular}




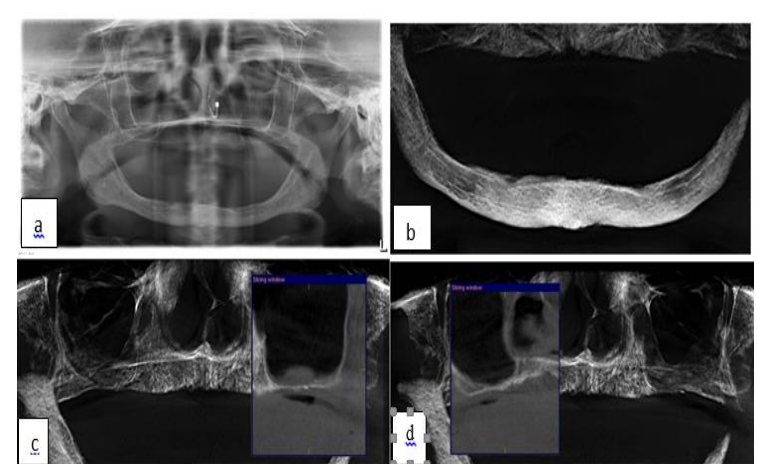

Fig. 1: Radiographs of the patient (a) Orthopantomo gram of jaws (b) CBCT shows the proximity of alveolar ridge and mandibular canal (c) CBCT magnified view of the left side of maxillary antral floor (d) CBCT magnified view of the right side of maxillary antral floor

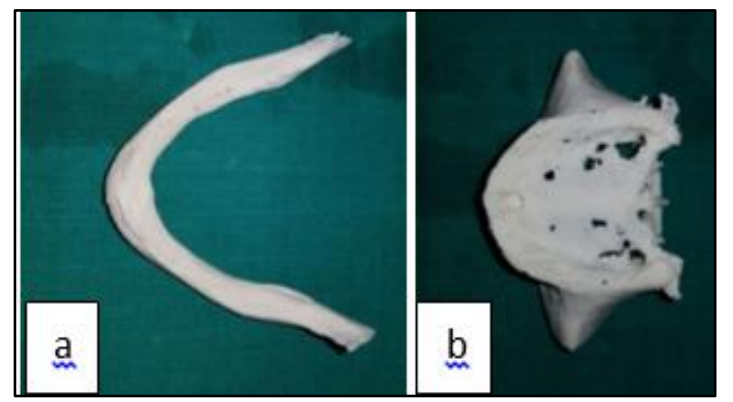

Fig. 2: Three dimensional models of (a) Lower jaw and (b) Upper jaw

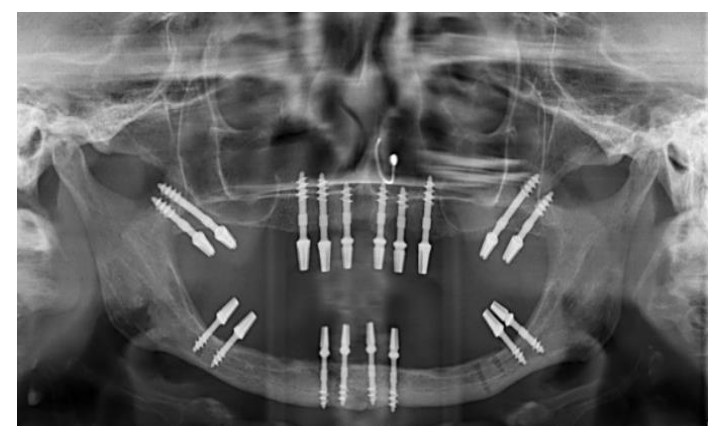

Fig. 3: Implants inserted into jaw bones in strategic locations

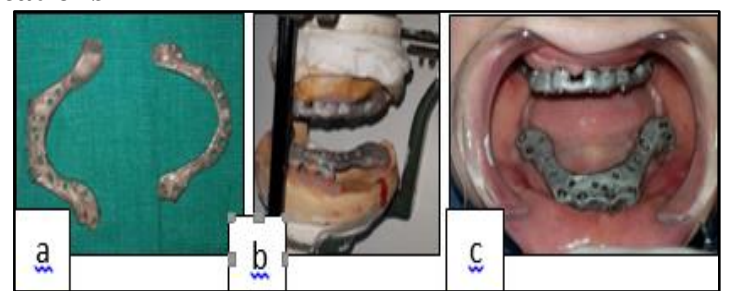

Fig. 4: Prosthetic works (a) Metal frame (b) on articulator (c) on patient's mouth

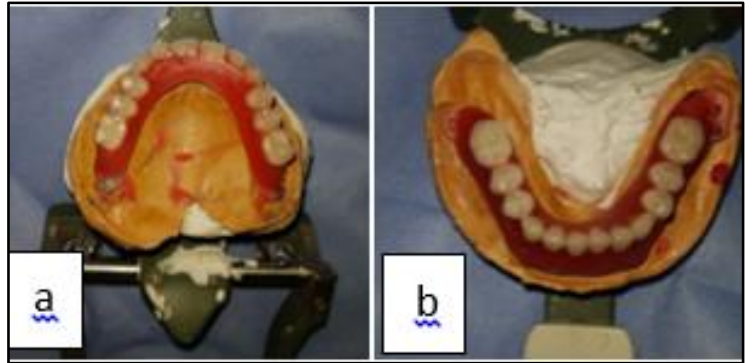

Fig. 5: Setting of teeth on wax rim (a) upper jaw (b) lower jaw

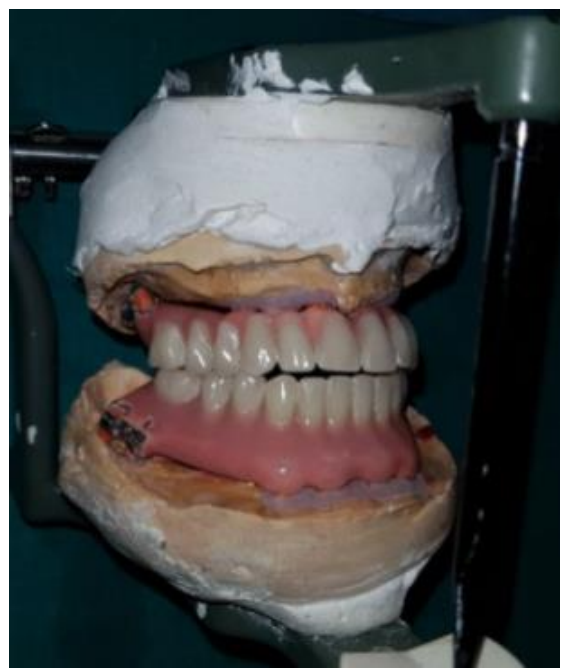

Fig. 6: Finished hybrid denture

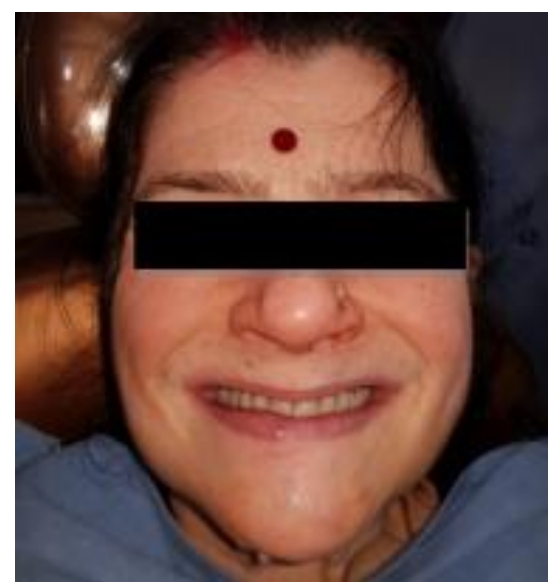

Fig. 7: Patient with implant retaineddenture

\section{Discussion}

Immediate loading of dental implants is a longawaited desire of both the patients and clinicians. ${ }^{7}$ This necessitates achieving a very strong primary stability of implants into bone. ${ }^{13}$ But conventional placement of implants into deficient spongy alveolar bone does not give rise to such primary stability in most of the time and, therefore, immediate loading is far beyond the dream. ${ }^{9}, 10$ Such situation is commonly managed through high precision techniques of vertical ridge augmentation with bone grafting with or without sinus 
lifting. Utilization of barrier membranes and alloplastic bone graft substitute biomaterials for ridge expansion are very common. Some prefer to use autogenous bone graft subjecting patients into another wound and morbidity. A good hand of expertise is in need of for such precision operation. The whole procedure of augmentation is a costly affair with uncertainty of success rate. A very highly atrophy of jaws may not be even managed by such expensive and cumbersome surgical procedure. ${ }^{8}$ Thus these patients are left behind and often advised not to go for implants and labelled as not restorable with conventional implant procedure. . $^{8,9,10}$ Therefore, conventional implant system poses lot of limitations which strategic implant system is able to overcome. ${ }^{11}$

The load bearing capacity of cortical bone is many times higher than that of cancellous bone; it is also strong, remains infection-free and lacks resorption. ${ }^{9}$ Screwable (not screw-type) basal and cortical implants (BECESTM, BCS ${ }^{\mathrm{TM}} \& \mathrm{KOC}^{\mathrm{TM}}$ ) have been developed with the idea of engagement into this strong bone overlooking the nature and amount of cancellous bone in the ridge. ${ }^{8}$ These are easily inserted in flapless procedure into cortical and basal bone either through extraction socket or into the highly mineralized part of basal bone yielding a very high primary stability. Trans-mucosal polished thin shaft does not allow bacteria to grow on it and hence, peri-implant diseases are largely prevented. ${ }^{8,9,10,11}$

In the present situation, with this high amount of bone resorption, the conventional removable complete mandibular and maxillary dentures were not suggested. Even the conventional implant system would require bone augmentation for the width and height throughout the length of the ridge for both the jaws with sinus lift; this would involve the delicate, sophisticated and expensive surgical interventions with unpredictable outcome. In addition to this, patient had to wait for healing time for both bone grafting and implant's osseo integration. This was not accepted by the patient in this case. She was happy to receive this implant system and enjoying chewing food and speech. Psychologically, she gained confidence in her life.

Acknowledgement: The authors gratefully acknowledge the masterly guidance and advice of Dr. Vivek Gaur of Delhi, an eminent implantologist of basal and cortical implants, for planning and surgery of the above case.

\section{Conflict of interest: Nil}

\section{Financial support: Nil}

\section{References}

1. Branemark PI, Adell R, Breine U, Hansson BO, Lindstrom J, Ohlsson A. Intra-osseous anchorage of dental prostheses. I. Experimental studies. Scand J Plast Reconstr Surg. 1969;3:81-100.

2. Adell R, Lekhholm U, Rockler B, Branemark PI. A 15year study of osseointegrated implants in the treatment of the edentulous jaw. Int J Oral Surg. 1981;10:387-416.

3. Araujo MG, Lindhe J. Dimensional ridge alterations following tooth extraction. An experimental study in the dogs. J Clin Periodontol. 2005;32:212-18.

4. Becker W, Becker BE. Guided tissue regeneration for implants placed into extraction sockets and for dehiscences: surgical techniques and case reports. Int $\mathbf{J}$ Periodentics Restorative Dent. 1990:10:376-391.

5. Bhar S, Badr J, Raviprakash BS, Das G,Pal TK. A study on sinus lifting on goat cadaver: In search of a teaching model. J of Indian Dent Assoc, 2017;11(6):23-28.

6. Barzilay I. Immediate implants: their current status. Int $\mathbf{J}$ Prosthodont 1993;6;169-175.

7. Buser D, Chappuis V, Belser UC, Chen S. Implant placement post extraction in esthetic single tooth sites: when immediate, when early, when late. Periodontology. 2000-2017;73:84-102.

8. Ihde $\mathrm{S}$ and IhdeA : Introduction into the work with the strategic implant ( $2^{\text {nd }}$ edition $)$. International Implant Foundation Publication. Munich Germany. 2018.

9. Ihde $S$ and IhdeA : Immediate Loading ( $2^{\text {nd }}$ edition). International Implant Foundation Publication. Munich Germany. 2012.

10. Ihde S: Principles of BOI. Heidelberg, Springer, Germany. 2005.

11. Ihde S: Comparison of basal and crestal implants and their modes of application. Smile Dental Journal, Vol. 4 , Issue 1, 2009.

12. Ihde $\mathrm{S}$ and IhdeA: Cookbook Mastication ( $2^{\text {nd }}$ edition). International Foundation Publishing. Munich, Germany. 2016.

13. Ihde S, Ihde A, Lysenko V, Konstantinovic VS, Palka: New systematic terminology of cortical bone areas for osseo-fixated implants in Strategic Oral Implantology. $J$. J Anatomy. 2016;1(2)1-9.

How to cite this article: Pal T. K, Mahata A, Das G. Severely atrophied jaws: A case report of full mouth rehabilitation using basal and cortical implants. Int J Periodontol Implantol. 2018;3(4):143146. 